\title{
Prevalência de inflamações reumatológicas crônicas em pacientes acometidos por chikungunya
}

\section{Prevalence of chronic rheumatological inflammations in patients affected by chikungunya}

\begin{abstract}
Milena Nunes Alves de Sousa ${ }^{1,2^{*}}$, Ruan Felipe Ferreira Tomé ${ }^{1}$, Damiana Gerleide Brito Valério ${ }^{1}$, Larissa Thais $\mathrm{Cruz}^{1}$, Thamires Pereira de Figueiredo ${ }^{1}$, Larissa de Araújo Batista Suarez ${ }^{2,3}$, Marriane Brito Macedo ${ }^{1}$, Petrônio Souto Gouveia Filho ${ }^{4}$
\end{abstract}

\begin{abstract}
RESUMO
O trabalho buscou estimar a prevalência de inflamações reumatológicas crônicas em pacientes acometidos por Chikungunya. Tratou-se de uma revisão sistemática da literatura com buscas na Literatura Latino-Americana e do Caribe em Ciências da Saúde, Medical Publications e Scientific Electronic Library Online. A pesquisa foi realizada em maio de 2020 e a estratégia de busca incluiu as combinações dos termos: "Chikungunya", "rheumatism", "post chikungunya" e "follow up". A maioria dos estudos (80\%) foi de coorte. Em relação ao local de realização, 30\% foram realizados na Colômbia. A prevalência das inflamações reumatológicas crônicas pós-infecção pelo vírus da Chikungunya variou de 25,3\% a $83,1 \%$. Há uma alta prevalência de lesões reumatológicas crônicas em pacientes, que não possuíam nenhuma doença reumatológica crônica preexistente e que foram infectados pelo vírus chikungunya. Além disso, grande parte dos estudos foi em países latino-americanos, possivelmente devido o fato de serem países tropicais.
\end{abstract}

Palavras-chave: Infecções por arbovirus; Vírus chikungunya; Doenças reumáticas; Prevalência; Epidemiologia.

\begin{abstract}
The work aimed to estimate the prevalence of chronic rheumatological inflammations in patients affected by Chikungunya. It involved a systematic review of the literature in the databases of Latin American and Caribbean Literature in Health Science, Medical Publications and Scientific Electronic Library Online. A survey was conducted in May 2020 and a search strategy included combinations of terms: "Chikungunya", "rheumatism", "post chikungunya" and "follow-up". Most studies (80\%) were cohort. Regarding the location of the study, 30\% were carried out in Colombia. The prevalence of chronic rheumatological inflammations after infection by the Chikungunya virus ranged from $25.3 \%$ to $83.1 \%$. There is a high prevalence of chronic rheumatological lesions in patients, who did not have any preexisting chronic rheumatological disease and who were infected by the chikungunya virus. In addition,
\end{abstract}

\footnotetext{
${ }^{1}$ Centro Universitário de Patos (UNIFIP), Patos, Paraíba, Brasil.

*E-mail: milenanunes@ fiponline.edu.br

${ }^{2}$ Faculdade São Francisco da Paraíba (FASP), Cajazeiras, Paraíba, Brasil.

${ }^{3}$ Universidade Católica do Pernambuco, Recife, Pernambuco, Brasil.

${ }^{4}$ Faculdade de Medicina do ABC (FMABC), São André, São Paulo, Brasil.
} 
most of the studies were done in Latin American countries, possibly due to the fact that they are poor countries.

Keywords: Arbovirus infections; Chikungunya virus; Rheumatic diseases; Prevalence; Epidemiology.

\section{INTRODUÇÃO}

A Chikungunya é uma arbovirose causada pelo vírus Chikungunya (CHIKV), o qual é transmitido aos humanos pela picada dos mosquitos Aedes aegypti e Aedes albopictus, apontados como os principais vetores. A propagação em todo o mundo deve-se, principalmente, à adaptação do mosquito a climas mais frios, ampliando sua distribuição geográfica, além das adaptações genéticas do vírus (ROUGERON et al., 2015; LIMA-CAMARA et al., 2016; HANDLER et al., 2017; DUARTE et al., 2018).

Entre 2013-2014, o CHIKV se propagou rapidamente nas Américas e em especial em países da América do Sul (PAULES; FAUCI, 2017). No final de 2013, o primeiro caso de transmissão de CHIKV nas Américas foi reportado em Saint Martin, região do Caribe. $\mathrm{N}$ final de 2014, países sul-americanos tais como Guiana Francesa, Venezuela, Colômbia, Suriname, Paraguai e Brasil já tinham notificado à circulação do vírus em seus territórios (CONTEVILLE et al., 2016). Historicamente, a letalidade relacionada ao vírus é baixa (BRASIL, 2018).

As manifestações clínicas são variáveis e compreendem desde formas assintomáticas ou oligossintomáticas até uma doença mais grave e potencialmente fatal (DAS et al., 2010). A tríade clássica composta por febre alta e abrupta, dor lombar e artralgia está presente em aproximadamente 92\% dos pacientes diagnosticados com Chikungunya (HANDLER et al., 2017).

Outras manifestações clínicas evidenciadas são mialgia, cefaleia, exantema e, em raros casos, alterações neurológicas. O período de incubação não difere com a idade, sendo, em média, de dois a sete dias (variação de um a 12 dias) (HANDLER et al., 2017). Cerca de $3 \%$ a $25 \%$ das pessoas com anticorpos para o CHIKV no soro têm infecções assintomáticas (STAPLES et al., 2009).

O diagnóstico laboratorial pode ser realizado por reação de cadeia polimerase transcriptase reversa (RT-PCR), ensaio imunoenzimático (ELISA) e detecção de imunoglobulina M (IgM) (SINGH et al., 2014; KUMAR et al., 2017). O tratamento da 
doença na fase aguda em adultos é principalmente de suporte (CABRAL-CASTRO et al., 2016), a base de fluidos, analgésicos e anti-inflamatórios não esteroides (AINES) para controle da febre e das dores articulares (ROBIN et al., 2016).

Normalmente, as lesões de pele são auto resolutivas. A terapêutica para artrite crônica secundária à febre de Chikungunya possui evidências limitadas na literatura (WAYMOUTH et al., 2013). Estudos citam a utilização de corticosteroides para os casos refratários ao uso de AINES ou mesmo quando esses são contraindicados (WAYMOUTH et al., 2013; CUNHA; SANTOS; ARAÚJO, 2021).

Além de não haver um tratamento eficaz para essas doenças reumatológicas crônicas secundárias ao agravo citado, dados quanto à sua prevalência são escassos. Dessa forma, o objetivo do presente estudo foi realizar uma revisão sistemática em estudos observacionais disponíveis para estimar a prevalência de inflamações reumatológicas crônicas em pacientes acometidos por Chikungunya.

\section{MATERIAL E MÉTODOS}

\section{Protocolo}

Esta revisão sistemática foi conduzida de acordo com o protocolo preconizado pelo Preferred Reporting Items for Systematic Reviews and Meta-Analyses (PRISMA) (MOHER et al., 2009).

\section{Desenho do estudo e critérios de elegibilidade}

Este estudo de revisão sistemática objetivou responder a seguinte questão clínica: "Qual a prevalência de inflamações reumatológicas crônicas em pacientes acometidos por Chikungunya?".

Foram incluídos estudos observacionais caso-controle, de coorte e transversais, sem restrição de ano, nos idiomas inglês, espanhol e português. Os critérios de exclusão foram: estudos não relacionados ao tema; artigos de revisão, cartas ao editor / editoriais, opiniões pessoais, livros / capítulos de livros, livros didáticos, relatórios, resumos de conferências e patentes. 
Fontes de informações e pesquisa e seleção dos estudos

Foram utilizadas as bases de dados Literatura Latino-Americana e do Caribe em Ciências da Saúde (LILACS), Medical Publications (PubMed)/ Medical Literature Analysis and Retrieval System Online (MEDLINE), incluindo publicações eletrônicas ahead of print e Scientific Electronic Library Online (SciELO), como fontes primárias de estudo.

O Medical Subject Headings (MeSH) foi utilizado para selecionar os descritores, usados com os operadores booleanos. A pesquisa bibliográfica foi realizada em maio de 2020 e a estratégia de busca incluiu as combinações dos termos: "Chikungunya", "rheumatism", "post chikungunya" e "follow up".

As pesquisas foram selecionadas em três momentos distintos. Na primeira fase, os revisores discutiram os critérios de elegibilidade e os aplicaram a uma amostra de $20 \%$ dos estudos recuperados para determinar a concordância entre examinadores. Esta concordância interexaminadores foi avaliada em dois momentos distintos, correspondendo às buscas iniciais (título e resumo) e, posteriormente, na busca definitiva (leitura completa dos artigos). Os títulos não relacionados ao tema e livros/capítulos de livros foram eliminados nesta etapa.

$\mathrm{Na}$ segunda fase, os revisores leram todos os resumos, independentemente e os resumos não relacionados ao tema foram eliminados. No terceiro estágio de busca, foram obtidos os artigos completos para serem lidos na íntegra e para observância aos critérios de elegibilidade. Em todas as fases, quando ambos os avaliadores discordaram, um terceiro revisor foi consultado para tomar uma decisão final. Os estudos rejeitados foram registrados separadamente, explicando-se as razões de sua exclusão.

\section{Coleta de dados}

Após identificação nas bases de dados $(\mathrm{n}=17.878)$, foram eliminados os estudos duplicados e for a do recorte temporal (2011-2019), em que foram excluídos 17.725. Um total de 153 trabalhos passaram pela análise criteriosa do título e resumo, levando a exclsuão de 135 artigos, mantendo-se, assim, para leitura do texto na íntegra, 18 artigos, dos quais apenas 10 artigos constituíram a amostra final. 
Após o delineamento amostral $(\mathrm{n}=10)$, dois autores analisaram os estudos e coletaram as seguintes informações dos estudos: Identificação do estudo (autor, ano, localização da pesquisa, tipo de estudo), características da amostra (número de pacientes em cada estudo), objetivo do estudo e acompanhamento. Qualquer desacordo entre os revisores foi resolvido com discussões aos pares e, quando ambos os revisores não puderam concordar, um o terceiro, considerado padrão-ouro, foi consultado para tomar a decisão final.

\section{RESULTADOS}

Esta revisão sistemática contemplou $80 \%$ de estudos de coorte, em que $30 \%$ foi procedente da Colômbia (Quadro 01).

Quadro 1 - Distribuição dos estudos selecionados para esta revisão

\begin{tabular}{|c|c|c|c|}
\hline Autores & País & Tipo de estudo & Nível de evidência \\
\hline Mathew et al. (2011) & Índia & Coorte & $2 \mathrm{~B}$ \\
\hline Kularatne et al. (2012) & Sri Lanka & Coorte & $2 \mathrm{~B}$ \\
\hline Javelle et al. (2015) & França & Coorte & $2 \mathrm{~B}$ \\
\hline $\begin{array}{l}\text { Rodriguez-Morales et } a l . \\
\text { (2016a) }\end{array}$ & Colômbia & Coorte & $2 \mathrm{~B}$ \\
\hline $\begin{array}{l}\text { Rodriguez-Morales et } a l \text {. } \\
\text { (2016b) }\end{array}$ & Colômbia & Coorte & $2 \mathrm{~B}$ \\
\hline Bouquillard et al. (2017) & França & Coorte & $2 \mathrm{~B}$ \\
\hline $\begin{array}{l}\text { Murillo-Zamora et } a l . \\
\text { (2017) }\end{array}$ & México & Coorte & $2 \mathrm{~B}$ \\
\hline Chang et al. (2018) & Colômbia & Coorte & $2 \mathrm{~B}$ \\
\hline O’Sullivan et al. (2018) & Jamaica & Transversal & $2 \mathrm{C}$ \\
\hline Panato et al. (2019) & Brasil & Transversal & $2 \mathrm{C}$ \\
\hline
\end{tabular}

Fonte: Pesquisa em bases de dados, 2020.

Um dos estudos teve amostra de mais de 1396 indivíduos. Contudo, apenas uma pesquisa teve um longo período de acompanhamento (seis anos). A prevalência das inflamações reumatológicas crônicas pós-infecção pelo vírus da Chikungunya variou de $25,3 \%$ a $83,1 \%$ (Quadro 02). 
Quadro 2 - Distribuição dos estudos selecionados para esta revisão quanto à amostra, período de acompanhamento e prevalência de distúrbios reumatológicos

\begin{tabular}{|c|c|c|c|}
\hline Autores & Amostra & $\begin{array}{c}\text { Período de } \\
\text { acompanhamento }\end{array}$ & $\begin{array}{l}\begin{array}{c}\text { Prevalência de distúrbios } \\
\text { reumatológicos }\end{array} \\
\end{array}$ \\
\hline Mathew et al. (2011) & 1396 & 15 meses & $31,3 \%$ \\
\hline Kularatne et al. (2012) & 512 & 36 meses & $46 \%$ \\
\hline Javelle et al. (2015) & 159 & 6 anos & $80 \%$ \\
\hline $\begin{array}{l}\begin{array}{l}\text { Rodriguez-Morales } \\
\text { (2016a) }\end{array} \\
\end{array}$ & 131 & 7 meses & $55,7 \%$ \\
\hline $\begin{array}{l}\text { Rodriguez-Morales et } \quad \text { al. } \\
\text { (2016b) }\end{array}$ & 283 & 26,1 semanas & $53,7 \%$ \\
\hline Bouquillard et al. (2017) & 307 & 32 meses & $83,1 \%$ \\
\hline Murillo-Zamora et al. (2017) & 136 & 6 meses & $41,9 \%$ \\
\hline Chang et al. (2018) & 485 & 20 meses & $25,3 \%$ \\
\hline O’Sullivan et al. (2018) & 306 & 12 meses & $\begin{array}{c}\text { Artralgia persistente }(30,6 \%) ; \\
\text { Artralgia severa }(27,5 \%)\end{array}$ \\
\hline Panato et al. (2019) & 130 & 12 meses & $38 \%$ \\
\hline
\end{tabular}

Fonte: Pesquisa em bases de dados, 2020.

\section{DISCUSSÃO}

O CHIKV foi isolado pela primeira vez na Tanzânia em 1952/53. A infecção ficou limitada à África e Ásia e uma mutação viral em 2005 permitiu o Aedes albopictus também se tornasse vetor do CHIKV, embora o mosquito Aedes aegypti seja o principal vetor (HANDLER et al., 2017). O mosquito Aedes albopictus habita regiões de clima temperado ao redor do mundo, incluindo regiões dos Estados Unidos da América (EUA), bem como países da Europa, África, Ásia e ilhas do Pacífico (DAS et al., 2010).

A distribuição mundial dos vetores permitiu a ocorrência de diversos surtos, inclusive, o mais grave já descrito, nas ilhas francesas de La Reunion, situadas no oceano pacífico, no ano de 2005/2006, em que 34\% da população (266.000/785.221) foi contaminada pelo CHIKV, com 203 óbitos (DAS et al., 2010). Outros surtos se seguiram, como o da Índia e em alguns países do sudeste asiático em 2006. Em 2007, alguns casos autóctones surgiram na Itália e em 2010 no sul da França (WAYMOUTH et al., 2013).

No Brasil, o primeiro caso autóctone foi relatado em 2014, em Oiapoque, Amapá. Em 2016, o Ministério da Saúde nacional reportou um total de 271.824 casos suspeitos de febre da chikungunya. No ano de 2017, foram registrados 185.854 casos suspeitos ao passo que em 2018, até o mês de Março, foram notificados 18.800 casos suspeitos da doença, com uma incidência de 9,1 casos/100 mil habitantes. Dos casos notificados até Março/2018, 9011 (47,9\%) em relação ao total do país pertence à região Centro-Oeste, $4861(25,9 \%)$. 
Embor a letalidade ainda serja considerada baixa, dados nacionais sugerem um aumento a partir de 2015 e 2016, tendo ocorrido respectivamente 6 e 156 mortes confirmadas por Chikungunya nestes anos (COLLUCCI, 2016). Para o autor, das 156 mortes ocorridas no Brasil, 150 foram na região Nordeste, e geralmente ocorreram entre 20 e 30 dias após a infecção pelo CHIKV.

Os indivíduos afetados desenvolvem abruptamente febre alta (85\%-100\%), normalmente acima de $38,9^{\circ} \mathrm{C}$ seguida em minutos a horas, por uma grave, e por vezes debilitante, artrite/artralgia que pode persistir ao final da doença, especialmente em indivíduos mais velhos com articulações já comprometidas, em um período de meses até mesmo anos (LIMA-CAMARA, 2016).

As dores nas articulações normalmente são simétricas e acomete inicialmente pequenas articulações das mãos, dos pés, punhos e tornozelos, podendo acometer também grandes articulações. Outros comemorativos da doença incluem mialgia (6093\%) (RITZ et al., 2015), cefaleia (40-85\%) (LIMA-CAMARA, 2016) e exantema, podendo chegar a $95 \%$ dos casos, principalmente do tipo maculopapular $(35-58,4 \%)$ (KUMAR et al., 2016). Este exantema comumente envolve tronco e extremidades, e geralmente causa prurido (80\%) (RITZ et al., 2015). No presente estudo, a persistência de inflamações reumatológicas crônicas foi de $25,3 \%$ a $83,1 \%$.

Segundo estudos realizados por Mathew et al. (2011) com 1396 pessoas e um acompanhamento por 15 meses, notou-se um acometimento de distúrbios reumatológicos de 31,3\%. Kularatne et al. (2012), reuniu 512 pessoas e as acompanhou por 36 meses tendo como resultado a porcentagem de $46 \%$ em relação a prevalência de doenças reumatológicas. Jovelle et al. (2015), reuniu um grupo de 159 pessoas acompanhando-as por 6 anos e observarm que a porcentagem das enfermidades reumatológicos alcançou 80\%. Rodriguez-Morales et al. (2016a), obteve em seu experimento uma taxa de 55,7\% em que observou por 7 meses 131 pessoas.

Rodriguez-Morales et al. (2016b) realizaram os mesmos experimentos observando 283 pessoas por 26,1 semanas e verificaram uma prevalência 53,7\% de distúrbios reumatológicos. Murillo-Zamora et al. (2017) observando 136 pessoas por 6 meses identificarm que 41,9\% apresentaram agravos reumáticos. Chang et al. (2018), em estudo com 485 pessoas acomapnhadas por 20 meses obteveram 25,3\% de prevalência. 
Os autores, a partir de uma amostra de 306 pessoas por um tempo de 12 meses, constaram que 30,6\% apresentaram artralgia persistente e 27,5\% severa (O'SULLIVAN et al., 2018). Panato et al. (2019), a partir de pesquisa com 130 pessoas as quais foram também acompanhadas por 12 meses, verificaram $38 \%$ de acometimentos reumatológicos. Bouquillard et al. (2017), a partir de investigação com 307 pessoas as quais foram acompanhadas por 32 meses, estimaram no grupo 83,1\% com doenças reumáticas.

Pelos achados desta revisão sistemática, o maior período de acompanhamento pode indicar maior prevalência de distúrbios reumatológicos, embora tenha havido excessões.

Em relação às manifestações neurológicas, estudo retrospectivo realizado na ilha de La Reunion com 30 crianças verificou a presença de encefalite $(n=12)$, convulsões febris $(n=10)$, síndrome meníngea $(n=4)$, e encefalopatia aguda $(n=4)$ (ROBIN et al., 2009). Estudo na Índia, em 2006, com 66 crianças com suspeita de infecção do Sistema Nervoso Central (SNC), 14\% tinham Chikungunya, diagnóstico positivo por RT-PCR, e apresentaram manifestações graves, incluindo estado de mal epilético e convulsões (RITZ et al., 2015). A análise bioquímica do líquido cerebrospinal foi realizada em pacientes com Chikungunya que desenvolveram convulsões, no entanto, em todos os casos os resultados foram normais (VALAMPARAMPIL et al., 2009).

A presença de febre com ou sem artralgia pode ocorrer em várias outras doenças. Também pode coexistir CHIKV com outras doenças infecciosas, como exemplos citam-se a dengue e a malaria. O diagnóstico diferencial de CHIKV com outras infecções (leptospirose, dengue, malária, outras infecções alphavirais) e, também, com artrite pós-infecciosa ou artrite reumatoide juvenil deve ser afastada pela clínica e por exames complementares (PANAMERICAN HEALTH ORGANIZATION, 2011), como ocorreu em todos os artigos selecionados para esta revisão.

A infecção pelo CHIKV ainda é uma doença muito prevalente no mundo, principalmente nos países tropicais, como observado nesta revisão. Além disso, com o passar do tempo o perfil de letalidade vem se modificando e as inflamações reumatológicas crônicas têm alta prevalência nos indivíduos acometidos. Dessa forma, torna-se necessária à intensificação de políticas públicas para controle da propagação dos vetores e de tratamentos com eficácia elevada nos casos graves e que as inflamações reumatológicas persistiram. 
Dentre as limitações, destaque para a pequena amostra de estudos, o que dificultou encontrar relações significativas a partir dos dados, já que as estatísticas exigem um tamanho amostral maior para garantir uma tendência representativa do grupo de pessoas acometidas pelo vírus da chikungunya. A falta de pesquisas anteriores sobre o tema abordado, também foi uma limitação do estudo.

\section{CONCLUSÃO}

Embora não se possa estabelecer uma relação causal, o estudo mostrou alta prevalência de lesões reumatológicas crônicas em pacientes que foram infectados pelo vírus chikungunya e os quais não possuíam nenhuma doença reumatológica crônica preexistente.

A maioria dos estudos era de países da América Latina, possivelmente devido ao fato de terem clima tropical. Apesar das pesquisas, em geral, adotarem um período de acompanhamento considerado satisfatório, mais investigações são necessárias para se estabelecer a relação causa-efeito entre a infecção pelo vírus Chikungunya e as inflamações reumatológicas crônicas.

\section{REFERÊNCIAS}

BRASIL. Ministério da Saúde. Secretaria de Vigilância em Saúde. Boletim Epidemiológico de 16. 2018. Disponível em: http://portalarquivos2.saude.gov.br/images/pdf/2018/abril/27/BE-2018-14-SE-12.pdf. Acesso em 23 mai 2020.

BOUQUILLARD, E. et al. Rheumatic manifestations associated with chikungunya virus infection: a study of 307 patients with 32-month follow-up (RHUMATOCHIK study). Joint Bone Spine, v. 85, n. 2, p. 207-210, 2018.

CABRAL-CASTRO, M. J. et al. Molecular and serological techniques to detect cocirculation of DENV, ZIKV and CHIKV in suspected dengue-like syndrome patients. Journal of Clinical Virology, v. 82, p. 108-111, 2016.

CHANG, A. Y. et al. Frequency of chronic joint pain following chikungunya virus infection: a colombian cohort study. Arthritis \& Rheumatology, v. 70, n. 4, p. 578$584,2018$.

COLLUCCI, C. Brazilian health authorities on alert after rise in deaths from chikungunya. BMJ, v. 355, p. 6360, 2016. 
CONTEVILLE, L. C. et al. Phylogenetic analyses of chikungunya virus among travelers in Rio de Janeiro. Brazil, 2014-2015. Memórias do Instituto Oswaldo Cruz, v. 111, n. 5, p. 347-348, 2016.

CUNHA, T. K. O.; SANTOS, E. V. L.; ARAÚJO, D. K. L. Tratamento com corticoide em pacientes com febre chikungunya: uma revisão integrativa da literatura. Brazilian Journal of Development, v. 7, n. 6, p. 55806-55816, 2021.

DAS, T. et al. Chikungunya fever: CNS infection and pathologies of a re-emerging arbovirus. Progress in neurobiology, v. 91, n. 2, p. 121-129, 2010.

DUARTE, R. S. et al. Sequelas da febre Chikungunya e sua interferência na qualidade de vida de indivíduos. Revista Brasileira de Qualidade de Vida (RBQV), v.10, p.1$14,2018$.

HANDLER, M. Z. et al. Chikungunya fever: an emerging viral infection threatening North America and Europe. International Journal of Dermatology, v. 56, n. 2, p. e19e25, 2017.

JAVELLE, E. et al. Specific management of post-chikungunya rheumatic disorders: a retrospective study of 159 cases in reunion island from 2006-2012. PLOS Neglected Tropical Diseases, v. 9, p. e0003603, 2015.

KULARATNE, S. A. et al. Epidemiology, clinical manifestations, and long-term outcomes of a major outbreak of chikungunya in a hamlet in Sri Lanka, in 2007: a longitudinal cohort study. Journal of Tropical Medicine, v. 2012, p. 639178, 2012.

KUMAR, R. et al. Cutaneous manifestations of chikungunya fever: Observations from an outbreak at a Tertiary Care Hospital in Southeast Rajasthan, India. Indian

Dermatology Online Journal, v. 8, p. 336-342, 2017.

LIMA-CAMARA, T. N. Emerging arboviruses and public health challenges in Brazil. Revista de Saúde Pública, v. 50, 2016.

MATHEW, A. J. et al. Rheumatic-musculoskeletal pain and disorders in a naive group of individuals 15 months following a chikungunya viral epidemic in south India: a population based observational study. International Journal of Clinical Practice, v. 65, p. 1306-1312, 2011.

MOHER, D. et al. Preferred reporting items for systematic reviews and meta-analyses: the PRISMA statement. PLoS Medicine, v. 6, n. 7, p.1000097.

MURILLO-ZAMORA, E. et al. Persistent arthralgia and related risks factors in laboratory-confirmed cases of Chikungunya virus infection in Mexico. Revista Panamericana de Salud Pública, v. 41, p. e72, 2017.

O'SULLIVAN, O. et al. Chikungunya virus infection-associated arthralgia in adult jamaicans post-outbreak. West Indian Medical Journal, v. 67, n. 3, p. 190-196, 2018. 
PAN AMERICAN HEALTH ORGANIZATION. Preparedness and Response for Chikungunya Virus: Introduction in the Americas; 2011.

PANATO, C. S. et al. Evaluation of functional disability after Chikungunya infection. Revista da Sociedade Brasileira de Medicina Tropical, v. 52, p. e20190112, 2019.

PAULES, C. I.; FAUCI, A. S. Emerging and reemerging infectious diseases: the dichotomy between acute outbreaks and chronic endemicity. JAMA, v. 317, n. 7, p. 691-692, 2017.

RITZ, N.; HUFNAGEL, M.; GÉRARDIN, P. Chikungunya in children. The Pediatric Infectious Disease Journal, v. 34, p. 789-791, 2015.

ROBIN, S. et al. Neurologic manifestations of pediatric chikungunya infection. Journal of Child Neurology, v. 23, p. 1028-1035, 2008.

ROBIN, S. et al. Severe bullous skin lesions associated with Chikungunya virus infection in small infants. European Journal of Pediatrics, v. 169, p. 67-72, 2010.

RODRÍGUEZ-MORALES, A. J. et al. Post-chikungunya chronic arthralgia: Results from a retrospective follow-up study of 131 cases in Tolima, Colombia. Travel Medicine and Infectious Disease, v. 14, n. 1, p. 58-59, $2016 \mathrm{a}$.

RODRIGUEZ-MORALES, A. J. Post-chikungunya chronic inflammatory rheumatism: Results from a retrospective follow-up study of 283 adult and child cases in La Virginia, Risaralda, Colombia. F1000Research, v. 5, p. 360, 2016 b.

ROUGERON, V. et al. Chikungunya, a paradigm of neglected tropical disease that emerged to be a new health global risk. Journal of Clinical Virology, v. 64, p. 144$152,2015$.

SINGH, N. et al. Vesiculobullous viral exanthem due to chikungunya in an infant. Indian Dermatology Online Journal, v. 5, n. 2, p. 119-120, 2014.

STAPLES, J. E.; BREIMAN, R. F.; POWERS, A. M. Chikungunya fever: an epidemiological review of a re-emerging infectious disease. Clinical Infectious Diseases, v. 49, n. 6, p. 942-948, 2009.

VALAMPARAMPIL, J. J. et al. Clinical profile of chikungunya in infants. The Indian Journal of Pediatrics, v. 76, p. 151-155, 2009. 
WAYMOUTH, H. E.; ZOUTMAN, D. E.; TOWHEED, T. E. Chikungunya-related arthritis: case report and review of the literature. In: Seminars in arthritis and rheumatism. WB Saunders, 2013. p. 273-278.

Recebido em: 15/10/2021

Aprovado em: 10/11/2021

Publicado em: 12/11/2021 